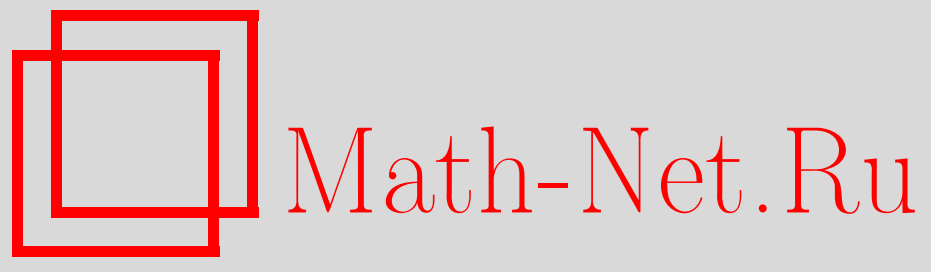

В. Крейг, К. Е. Вейн, Математические аспекты поверхностных волн на воде, УМН, 2007, том 62, выпуск 3, 95116

DOI: https://doi.org/10.4213/rm6759

Использование Общероссийского математического портала Math-Net.Ru подразумевает, что вы прочитали и согласны с пользовательским соглашением http://www .mathnet.ru/rus/agreement

Параметры загрузки:

IP: 54.210 .77 .194

26 апреля 2023 г., 13:09:41

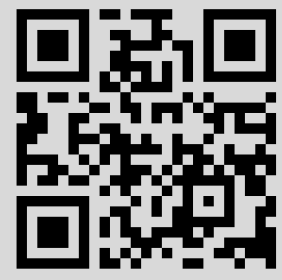




\title{
Математические аспекты поверхностных волн на воде
}

\author{
В. Крейг, К.Е. Вейн
}

Теория движения свободной поверхности воды всегда привлекала внимание исследователей. Она имеет глубокую историю как в чистой математике, так и в приложениях, связанных с хозяйственной деятельностью на море. Несмотря на значительные успехи в этой области (о некоторых из них можно было услышать на конференции по математической гидродинамике в институте им. В.А. Стеклова) и на постоянное пристальное внимание математического сообщества к этой тематике, остается много нерешенных фундаментальных проблем. Предстоит еще много сделать в области эволюции поверхностных волн на воде, приближения динамики этих волн модельными уравнениями и их использования в компьютерных экспериментах. Необходимо уточнить динамику взаимодействия волн, которая приводит к образованию бродячих волн в открытом океане. Предстоит построить теорию (отчасти вероятностную), описывающую волновые поля в больших водоемах с помощью усреднения "микроскопических" величин, которые по сути удовлетворяют кинетическим уравнениям движения. В данной заметке мы постарались обратить внимание на открытые проблемы и осветить некоторые направления современных исследований в этой сфере. Мы исходим из того, что появление новых аналитических методов и ранее неизвестных точек зрения сыграет важную роль в будущем развитии этой науки.

Библиография: 54 названия.

\section{СОДЕРЖАНИЕ}

1. Уравнения движения.................................... 96

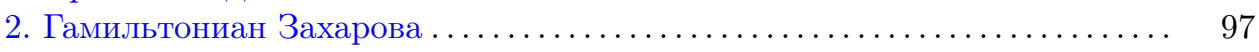

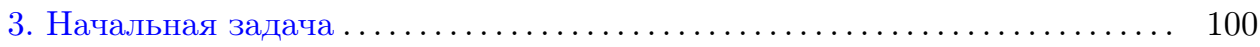

4. Длинные волны и модуляционные пределы . . . . . . . . . . . . . . 103

5. Бегущие волны . . . . . . . . . . . . . . . . . . . . . . . . . . . . . . . . . . . 104

6. Инвариантные структуры в фазовом пространстве ............... 107

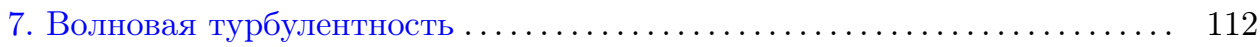

Список литературы ......................................... 114

Работа В. Крейга частично поддержана Канадской кафедральной исследовательской программой, грантом NSERC № 238452, а также грантом NSF № DMS-0070218. Работа K. Е. Вейна поддержана NSF. Численное моделирование для этого обзора выполнил P. Guyenne.

(C) В. КРейг, К. Е. ВЕйн, 2007 


\section{1. Уравнения движения}

В общей постановке поверхностные волны на воде связаны с динамикой жидкости, удовлетворяющей уравнениям движения Эйлера и заполняющей пространственно-временную область со свободной границей, которая движется под действием "объемных сил" земного притяжения. Кроме того, предполагается, что жидкость является несжимаемой, что весьма естественно для воды в нормальных природных условиях. Также считается, что в жидкости отсутствуют завихрения, что в общей постановке представляется уже более натянутым условием. Однако эти гипотезы приемлемы для описания распространения волн на поверхности воды в океане или в других водоемах, и они повсеместно используются в океанографии. Уравнения движения в эйлеровых координатах описывают поле скорости $\mathbf{u}(x, t)$, которое удовлетворяет уравнениям

$$
\nabla \cdot \mathbf{u}=0, \quad \nabla \times \mathbf{u}=0 .
$$

В силу двух этих условий, поле скорости можно представить в виде градиента гармонической функции:

$$
\mathbf{u}=\nabla \varphi, \quad \Delta \varphi=0 .
$$

Будем считать, что наша жидкость заполняет некоторую пространственновременную область, которая является подмножеством евклидова пространства $\Sigma \subseteq \mathbb{R}^{d+1}$, причем обычно выбирается $d=3$ или 2 . Хотя описанию подвергаются волны с широким распространением, типа цунами, для наших целей достаточно считать, что "земля" является плоской с пространственными координатами $\left\{(x, y): x \in \mathbb{R}^{d-1}, y \in \mathbb{R}\right\}$, а сила притяжения имеет вид $F=-g y$. Кроме того, предполагается, что занимаемый объем жидкости имеет неподвижную нижнюю поверхность (дно океана), которая задается формулой $\{(x, y): y=-b(x)\}$, и в классической постановке рассматривается плоская область, а именно предполагается, что функция $b(x)=h$ является постоянной. Считается, что на дне, как и возле любых твердых участков границы жидкости, течение не имеет нормальной составляющей:

$$
\mathbf{u} \cdot N=0,
$$

а на само́й свободной границе ставятся два классических граничных условия, кинематическое условие и условие Бернулли. Для случая свободной поверхности, заданной графиком функции $\{y=\eta(x, t)\}$ (однако всем, кто занимается серфингом в Калифорнии, известно, что самый интересный случай исключается таким ограничением), эти два граничных условия выражаются уравнениями

$$
\partial_{t} \eta=\partial_{y} \varphi-\partial_{x} \eta \cdot \partial_{x} \varphi, \quad \partial_{t} \varphi=-g \eta-\frac{1}{2}|\nabla \varphi|^{2} .
$$

Если учитывать эффект поверхностного натяжения, то изменится второе уравнение - добавится член, пропорциональный средней кривизне свободной поверхности. Случай, когда поверхность не сводится к графику функции, также можно рассматривать, выбрав другие координаты. Задача с начальными условиями заключается в нахождении решения $(\eta(x, t), \varphi(x, y, t))$ в области $S(\eta(\cdot, t))=\left\{(x, y): x \in \mathbb{R}^{d-1},-b(x)<y<\eta(x, t)\right\}$ на временно́м интервале $t \in[-T, T]$ для заданных начальных данных $\left(\eta_{0}(x), \varphi_{0}(x, y)\right)$ при условии, что $\varphi_{0}(x, y)$ является гармонической функцией в исходной области $S\left(\eta_{0}\right)$, которая описывается свободной поверхностью $\left\{y=\eta_{0}(x)\right\}$.

Очевидно, что при условиях (1.3) на свободную поверхность константа, задающая ускорение свободного падения, возникает в члене более низкого порядка, 
и поэтому ее влияние в эволюции решений должно быть также не столь значительным. Однако любой, кто держал в руках ведро с водой, вам скажет, что разница в знаке $g$ значительно влияет на динамику жидкости. Оказывается, что задача (1.3) является гиперболической, но с кратными характеристиками. Это отражает тот факт, что знак члена низкого порядка существенно влияет на поведение решений начальной задачи.

\section{2. Гамильтониан Захарова}

В замечательной работе В. Е. Захарова [1] задача о поверхностных волнах на воде была переформулирована в виде гамильтоновой системы с бесконечным числом степеней свободы, подобно тому как это делается для уравнения $\mathrm{KdV}$, нелинейного уравнения Шрёдингера и нелинейных волновых уравнений. Легко догадаться, что гамильтониан имеет вид функционала полной энергии:

$$
H=\int_{\mathbb{R}^{d-1}} \int_{-b(x)}^{\eta(x)} \frac{1}{2}|\nabla \varphi(x, y)|^{2} d y d x+\int_{\mathbb{R}^{d-1}} \frac{g}{2} \eta^{2}(x) d x .
$$

Более тонкий вопрос заключается в выборе сопряженных канонических переменных. Утверждение Захарова состоит в том, что величины $\eta(x)$ и $\xi(x):=$ $\varphi(x, \eta(x))$ являются подходящими переменными, с помощью которых задачу о волнах на свободной поверхности воды можно записать в виде канонических уравнений Гамильтона

$$
\partial_{t}\left(\begin{array}{l}
\eta \\
\xi
\end{array}\right)=J \operatorname{grad}_{(\eta, \xi)} H(\eta, \xi), \quad \text { где } \quad J=\left(\begin{array}{cc}
0 & I \\
-I & 0
\end{array}\right) .
$$

В силу потенциальной природы течения, достаточно знать область, задаваемую функцией $\eta(x, t)$, и граничные значения потенциала скорости $\xi(x, t)$ в любой конкретный момент времени $t$, для того чтобы восстановить течение во всем объеме в этот момент времени; в самом деле, имея (гладкую) область, заданную функцией $\eta$, достаточно знать граничные данные $\xi(x, t)$, чтобы определить потенциал скорости $\varphi(x, y)$, который является гармонической функцией на объеме жидкости $S(\eta)$, и, следовательно, данные $(\eta, \xi)$ задают поле скорости жидкости $\mathbf{u}(x, y, t)=\nabla \varphi(x, y)$ по всему объему в момент времени $t$.

Формализуя приведенное выше высказывание, определим оператор Дирихле-Неймана для объема жидкости $S(\eta)$ с помощью соотношения

$$
\xi(x) \mapsto \varphi(x, y) \mapsto N \cdot(\nabla \varphi)(x, \eta(x)) d S_{\eta}:=G(\eta) \xi(x) d x .
$$

Этот оператор является линейным по $\xi(x)$, но в целом он нелинеен и вполне нелокален в своей зависимости от объема жидкости, который задается свободной поверхностью $\eta(x)$. Введение нормы обеспечивает самосопряженность оператора $G(\eta)$ (с подходящей областью определения) на $L^{2}\left(\mathbb{R}^{d-1}\right)$. Переписав гамильтониан (2.1) и использовав этот оператор (см. [2]), получаем

$$
H(\eta, \xi)=\int_{\mathbb{R}^{d-1}}\left(\frac{1}{2} \xi(x) G(\eta) \xi(x)+\frac{g}{2} \eta^{2}(x)\right) d x .
$$

Канонические гамильтоновы уравнения (2.2) эквивалентны уравнениям движения с условиями свободной границы (1.3). В самом деле, первое уравнение (1.3) является прямым следствием определения оператора Дирихле-Неймана; второе уравнение следует из вычислений, которые тесно связаны с вариационной формулой Адамара. 
Классические консервативные величины для задачи о волнах на воде выражаются в этих переменных в виде интегралов. Действительно, добавленная масса точки $(\eta, \xi)$ в фазовом пространстве задается формулой

$$
M(\eta, \xi):=\int_{\mathbb{R}^{d-1}} \eta(x) d x,
$$

а в случае плоского дна, $b(x)=h$, горизонтальный момент (или импульс) по направлению $j$-й координаты имеет вид

$$
I_{j}(\eta, \xi):=\int_{\mathbb{R}^{d-1}} \xi(x) \partial_{x_{j}} \eta(x) d x .
$$

Определим скобку Пуассона по формуле

$$
\{F, G\}:=\int_{\mathbb{R}^{d-1}}\left(\operatorname{grad}_{\eta} F \operatorname{grad}_{\xi} G-\operatorname{grad}_{\xi} F \operatorname{grad}_{\eta} G\right) d x .
$$

Тогда свойство сохранения для заданных величин состоит в том, что они перестановочны с гамильтонианом $H$ по скобке Пуассона; можно проверить для обеих величин, что

$$
\{H, I\}=0, \quad\{H, M\}=0 .
$$

На самом деле, канонические переменные можно выбирать согласно "первому принципу" механики. Для кривой $\eta(\cdot, t)$, заданной в конфигурационном пространстве, лагранжиан, ассоциированный с (2.1), определяется как разность между кинетической и потенциальной энергией, точнее,

$$
L:=L(\eta, \dot{\eta})=\int_{\mathbb{R}^{d-1}} \int_{-b(x)}^{\eta(x)} \frac{1}{2}|\nabla \varphi(x, y)|^{2} d y d x-\int_{\mathbb{R}^{d-1}} \frac{g}{2} \eta^{2}(x) d x .
$$

Кинетическую энергию можно полностью переписать в терминах переменных $(\eta, \dot{\eta})$ с помощью оператора Дирихле-Неймана и кинематических уравнений $\dot{\eta}=G(\eta) \xi$, взятых из (1.3):

$$
L(\eta, \dot{\eta})=\int_{\mathbb{R}^{d-1}}\left(\frac{1}{2} \dot{\eta} G^{-1}(\eta) \dot{\eta}-\frac{g}{2} \eta^{2}\right) d x .
$$

Тогда преобразование Лежандра задает координаты, которые являются канонически сопряженными к $\eta$. В самом деле,

$$
\delta_{\dot{\eta}} L=G^{-1}(\eta) \dot{\eta},
$$

откуда следует, что $\xi(x)=\varphi(x, \eta(x))$ служит подходящим выбором, что и было сделано выше.

Принцип наименьшего действия Лагранжа для задачи о поверхностных волнах был независимо введен в работе [3] для функционала действия

$$
\begin{gathered}
A=\int_{t_{1}}^{t_{2}} L^{+}(\varphi, \eta) d t \\
L^{+}=-\int_{\mathbb{R}^{d-1}} \int_{-b(x)}^{\eta(x, t)}\left(\partial_{t} \varphi+\frac{1}{2}|\nabla \varphi|^{2}+g y\right) d y d x .
\end{gathered}
$$

Лагранжиан $L^{+}$отличается от заданного выше функционала $L$ полной производной по времени, и, следовательно, он соответствует той же формулировке 
задачи о поверхностных волнах как аналога бесконечномерной системы классической механики. Чтобы увидеть это, запишем

$$
\begin{aligned}
L^{+}=- & \frac{d}{d t}\left(\int_{\mathbb{R}^{d-1}} \int_{-b(x)}^{\eta(x, t)} \varphi(x, y, t) d y d x\right)+\int_{\mathbb{R}^{d-1}} \partial_{t} \eta \varphi(x, \eta(x, t), t) d x \\
& -\int_{\mathbb{R}^{d-1}} \int_{-b(x)}^{\eta(x, t)} \frac{1}{2}|\nabla \varphi(x, y, t)|^{2} d y d x-\int_{\mathbb{R}^{d-1}} \int_{-b(x)}^{\eta(x, t)} g y d y d x .
\end{aligned}
$$

Используя оператор Дирихле-Неймана и интегрируя по переменной $y$, получаем, что правая часть (2.13) равна

$$
\begin{gathered}
-\frac{d}{d t}\left(\int_{\mathbb{R}^{d-1}} \int_{-b(x)}^{\eta(x)} \varphi(x, y, t) d y d x\right)+\int_{\mathbb{R}^{d-1}} \dot{\eta} \xi d x \\
-\int_{\mathbb{R}^{d-1}}\left(\frac{1}{2} \dot{\eta} G^{-1}(\eta) \dot{\eta}+\frac{g}{2} \eta^{2}\right) d x+C .
\end{gathered}
$$

Отбрасывая константу $C$, которая не относится к динамике, и учитывая кинематическое условие (1.3), находим

$$
L^{+}=\int_{\mathbb{R}^{d-1}}\left(\frac{1}{2} \dot{\eta} G^{-1}(\eta) \dot{\eta}-\frac{g}{2} \eta^{2}\right) d x-\frac{d}{d t}\left(\int_{\mathbb{R}^{d-1}} \int_{-b(x)}^{\eta(x, t)} \varphi(x, y, t) d y d x\right)
$$

что действительно отличается от нашего выражения для $L$ наличием полной производной по времени.

В случае покоящейся поверхности $\eta=0$, если низ объема жидкости является плоским, то для оператора Дирихле-Неймана имеется явное выражение. Используя преобразование Фурье, можно записать

$$
G(0) \xi(x)=\frac{1}{2 \pi^{(d-1) / 2}} \int_{\mathbb{R}^{d-1}} e^{i k \cdot x}|k| \operatorname{th}(h|k|) \widehat{\xi}(k) d k=|D| \operatorname{th}(h|D|) \xi(x),
$$

где, как обычно, $D:=-i \partial_{x}$. Применяя это выражение в гамильтониане $(2.4)$ и беря квадратичную часть результата (линеаризующие уравнения около нулевого решения), получаем классические дисперсионные соотношения

$$
\omega^{2}(k)=g|k| \operatorname{th}(h|k|) .
$$

В итоге, фазовая и групповая скорости решений имеют вид

$$
c_{p}(k)=\sqrt{\frac{g \operatorname{th}(h|k|)}{|k|}} \frac{k}{|k|}, \quad c_{g}(k)=\partial_{k} \sqrt{g|k| \operatorname{th}(h|k|)} .
$$

Предел при больших $|k| \rightarrow+\infty$ групповой скорости дает скорость характеристик. В случае поверхностных волн на воде имеем

$$
\lim _{|k| \rightarrow+\infty} c_{g}(k)=0
$$

Это означает, что характеристики являются вещественными для любого направления $k /|k|$. Этот факт известен любому пловцу; на поверхности воды короткие волны движутся медленнее, чем длинные. Если не принимать в расчет дополнительные эффекты, например явления капиллярности, то больше ничто 
не влияет на скорость волн, и верхние пределы волновых чисел $c_{g}$ и $c_{p}$ равны нулю. В этом заключается явление гиперболичности с кратными характеристиками, которое было упомянуто в первом разделе; оно служит математическим объяснением чувствительности начальной задачи к знаку $g$ в подчиненном символе в правой части уравнения (1.3). С другой стороны, поверхностное натяжение имеет дисперсивный эффект, который во многих случаях (в некоторой степени) регуляризует задачу.

\section{3. Начальная задача}

Имеет смысл начать обсуждение с начальной задачи, поскольку (1.3) и эквивалентная ей система (2.2) записаны в таком виде. Мы будем обращаться с решением, которое в момент времени $t=0$ находится в состоянии $\left(\eta_{0}(x), \xi_{0}(x)\right)$, используя поток $\Phi_{t}\left(\eta_{0}, \xi_{0}\right)$, действующий (по крайней мере для последнего уравнения) в фазовом пространстве, требующем определения. Сразу сформулируем интересующие нас основные вопросы: существует ли введенный поток, какое пространство функций следует взять в качестве фазового пространства, как долго по времени будет существовать решение, следует ли ограничиться начальными данными в малой окрестности нулевой точки, или же можно брать любые начальные данные и строить решения, глобальные по времени.

Строгое математическое исследование начальной задачи при $d=2$ было инициировано в [4], где в качестве фазового пространства использовалось пространство аналитических функций, и в [5], где рассматривались функции, имеющие конечные нормы Соболева. Независимое исследование в пространстве аналитических функций было опубликовано в [6]. В работах [7]-[9] получены первые результаты в случае трехмерной задачи $(d=3)$ снова для аналитических функций, а статья [10] также содержит результаты для аналитического случая как предела сопряженной задачи с верхней жидкостью нулевой плотности.

Начальная задача, поставленная в категории соболевских пространств, является более важным случаем, поскольку, помимо прочего, большинство результатов в категории аналитических функций являются нечувствительными к знаку ускорения свободного падения $g$. Результаты статьи [5] были обобщены в [11] для переменной топографии дна и в [12] для задачи с поверхностным натяжением, а также в работах [13], [14], [15] в части существования на больших интервалах времени и для масштабных режимов уравнений Буссинеска и $\mathrm{KdV}$. Среди более поздних результатов упомянем работу [16] о существовании решения для начального объема жидкости достаточно произвольной формы (конечно, на коротком интервале времени), а также работы [17] и [18], где наметился прогресс для начальной задачи в пространстве размерности три (и даже больше).

ПроБЛЕмА 1. Представляется разумным доказать, что решение существует глобально по времени, по крайней мере для достаточно мальх начальнъх условий. Другими словами, поток $\Phi_{t}(\eta, \xi)$ должен существовать при всех $t \in \mathbb{R}$ в шаре $B_{r}(0)$ (с, возможно малым, радиусом $r$ и с иентром в нуле) в подходящем функциональном пространстве.

Ни один из перечисленных выше результатов не является глобальным по времени, однако известно, что глобальные решения существуют, например имеются периодические бегущие волны, построенные в работах [19], [20] и [21], или уединенные волны из работ [22] и [23]. Были построены также примеры трехмерных (и при $d>3$ ) кратно-периодических решений. Различные подходы к 
начальной задаче используют разные системы координат, в том числе лагранжевы координаты [5], координаты, получаемые при конформных отображениях объема жидкости $(d=2,[19]$ и [6]), и эйлеровы координаты [18]. К настоящему моменту последняя упомянутая работа ближе всего к рассматриваемой проблеме. Выбор координат в значительной степени влияет на характер анализа. Исследование, основанное на гамильтоновой структуре уравнений движения, потребует тщательного анализа коммутаторов $\left[\partial_{x}^{\alpha}, G(\eta)\right]$. При наличии хорошо разработанной техники аналитических функций, а также теории канонических преобразований по аналогии с гамильтоновой механикой, представляется аномальным, что эти методы все еще не так глубоко применяются при изучении начальной задачи поверхностных волн на воде.

ПроБлема 2. Использовать координаты, в которых задача о волнах на воде имеет гамильтонову структуру, как важное направление исследования начальной задачи.

Например, можно использовать анализ нормальных форм Биркгофа с целью доказательства теорем существования на длинных или бесконечных временнь́х интервалах. Чтобы развить эту идею применительно к рассматриваемой проблеме при $d \geqslant 3$, потребуется найти преобразование нормальных форм, которое после применения к гамильтониану поверхностных волн (2.4) сократит кубические члены, после чего можно будет воспользоваться убывающими по времени оценками, которые дадут глобальные оценки для супремум-нормы решения. Иначе говоря, гамильтониан $H$ можно разложить в ряд Тейлора в окрестности стационарной точки $(\eta, \xi)=0$ :

$$
H(\eta, \xi)=H^{(2)}(\eta, \xi)+\sum_{j \geqslant 3} H^{(j)}(\eta, \xi),
$$

поскольку имеется параллельное разложение оператора Дирихле-Неймана

$$
G(\eta) \xi=G^{(0)}(\eta) \xi+\sum_{j \geqslant 1} G^{(j)}(\eta) \xi
$$

и, в частности, при $j \geqslant 3, H^{(j)}(\eta, \xi)=\frac{1}{2} \int \xi G^{(j)}(\eta) \xi d x$. Разумно искать преобразования $\tau:(\eta, \xi) \mapsto\left(\eta^{1}, \xi^{1}\right)$, которые устранят все существенные нелинейности в уравнениях движения. В силу гамильтоновой природы системы, представляются естественными поиски канонических преобразований, с помощью которых можно преобразовать гамильтониан к нормальной форме Биркгофа до некоторого фиксированного порядка $N$ :

$$
\left(H \circ \tau_{N}\right)\left(\eta^{N}, \xi^{N}\right)=H^{(2)}\left(\eta^{N}, \xi^{N}\right)+\sum_{3 \leqslant j \leqslant N} H_{\text {resonant }}^{(j)}\left(\eta^{N}, \xi^{N}\right)+R^{(N+1)} .
$$

Дело в том, что все резонансные члены коммутируют по Пуассону с $H^{(2)}$; действительно, $\left\{H^{(2)}, H_{\text {resonant }}^{(j)}\right\}=0$.

Для задачи поверхностных волн на воде, без учета поверхностного натяжения, было давно понято, что не существует нетривиальных резонансов порядка $j=3$, и поэтому можно было бы построить преобразование нормальных форм Биркгофа $\tau_{3}$ такое, что кубический член в итоговом гамильтониане будет отсутствовать:

$$
H \circ \tau_{3}=H^{(2)}+R^{(4)} .
$$


Однако к настоящему времени аналитические свойства этого преобразования не установлены даже для случая $d=2$. Тем не менее, если предположить, что эту процедуру можно выполнить в подходящем функциональном пространстве с хорошими свойствами ограниченности преобразования $\tau_{3}$ и его обратного, то, возможно, получатся хорошие следствия и для начальной задачи. После преобразования нормальных форм Биркгофа при $N=3$ уравнения движения $(2.2)$ станут линейными плюс кубические нелинейные члены, а не линейными плюс квадратичные члены. Тогда энергетические неравенства дадут оценки решений, скорость роста которых будет квадратичной относительно неизвестных функций $(\eta, \xi)$. Ожидается, что решения лианеризованной задачи

$$
\partial_{t} \eta=\operatorname{grad}_{\xi} H^{(2)}, \quad \partial_{t} \xi=-\operatorname{grad}_{\eta} H^{(2)}
$$

будут удовлетворять оценкам убывания вида

$$
\|(\eta(\cdot, t), \xi(\cdot, t))\|_{L^{\infty}} \leqslant \frac{C}{\langle t\rangle^{(d-1) / 2}},
$$

для которых квадрат правой части интегрируем по $t$ при $d \geqslant 3$. Возможно, в этом заключается ключ к доказательству глобального существования гладких решений, по крайней мере в малой окрестности нуля подходящего фазового функционального пространства. Выражение в правой части (3.6) не интегрируемо по $t$ при $d=2$, но похожие оценки и аналогичные рассуждения могут привести к теоремам существования на достаточно длинных временны́х интервалах. Неравенство Стрихарца, подходящее для задачи о поверхностных волнах, может стать альтернативой использованию точного утверждения вида (3.6).

\section{ПроБЛЕмА 3. Как происходит разрушение решений?}

Существует много разновидностей данного вопроса, включая следующую: "Чему равен наименьший показатель пространства Соболева $H^{s}$, при котором можно предъявить теорему существования локального по времени решения?" Или можно спросить: "При каком $\alpha$ верно, что если априори известно неравенство $\sup _{[-T, T]}\|(\eta(\cdot, t), \xi(\cdot, t))\|_{C^{\alpha}}<+\infty$ и условие $\left(\eta_{0}, \xi_{0}\right) \in C^{\infty}$, то решение, на самом деле, принадлежит классу $C^{\infty}$ на интервале $[-T, T]$ ?" В настоящее время известен следующий ответ на первый вопрос: можно взять любой показатель $s>4$, но будет неправильно утверждать, что решение перестанет существовать после $t=T$, поскольку оно больше не лежит в $H^{4+}$. Правильнее будет сказать, что решение перестанет существовать, поскольку "кривизна поверхности расходится в некоторой точке", или сформулировать близкое геометрическое и/или физическое утверждение. Кроме того, хотя считается "очевидным", что решения не существуют очень долго после опрокидывания (т.е. после того как наклон поверхности воды становится бесконечным и он перестанет быть графиком гладкой функции $\eta(x, t))$, строгое математическое доказательство этого факта отсутствует. Нет даже специальных примеров, типа самоподобных решений, которые подтвердили бы это интуитивно верное утверждение.

Задача со свободной границей в невесомости является близкой проблемой, которая, однако, оказалась еще более трудной, чем в случае с гравитацией, где она служит восстанавливающей силой. Было доказано, что условие несжимаемости является достаточным для корректности начальной задачи, локальной по времени в случае конфигураций, близких к стационарным течениям; это сделано в работах [24] и [25]. Если течение находится в интенсивном движении, 
например если оно быстро вращается, то соответствующая начальная задача является некорректной; см. [26].

\section{4. Длинные волны и модуляционные пределы}

Нелинейные уравнения, которые используются при моделировании волн на воде, видимо, еще более известны, чем полная система уравнений Эйлера. Эти модели включают уравнения Буссинеска, уравнение Кортевега-де Фриса $(\mathrm{KdV})$, нелинейное кубическое уравнение Шрёдингера, систему Дэви-Стюартсона, уравнения Кадомцева-Петвиашвили (KP), а также их аналоги более высокого порядка, которые могут содержать дополнительные члены. Орбиты некоторых из этих систем в бесконечномерных фазовых пространствах хорошо изучены, так как они являются полностью интегрируемыми гамильтоновыми динамическими системами. Кроме того, имеется множество алгебраических структур, связанных с интегралами этих уравнений, в то время как полная система уравнений волн на воде имеет в общем случае существенно меньше интегралов движения и постоянных структур в соответствующем фазовом пространстве (см. [27]). В настоящей статье не будут освещаться результаты, относящиеся непосредственно к этим уравнениям, но будет обращено внимание на математическое обоснование их использования при приближении решений уравнений Эйлера (1.3).

В работе [4] и [6] рассматривалась задача со свободной поверхностью на мелкой воде в соответствующем масштабном пределе для случая $d=2$. Более поздняя работа [28] обращалась к разложению Фридрихса, а в статьях [13] и [29] изучались масштабные режимы уравнений Буссинеска и $\mathrm{KdV}$. Отметим, что медленное время, которое является характеристикой предельных масштабных режимов Буссинеска и $\mathrm{KdV}$, указывает на уместность изучения начальной задачи на длинных временных интервалах для получения связи между решениями уравнений Эйлера и решениями подходящего предела длинных волн. Эта тема получила новое развитие в работе [14], в которой рассматривались вопросы взаимодействия уединенных волн с учетом сил поверхностного натяжения. Обобщение результатов этой работы для более высоких порядков имеется в статье [30]. Все перечисленные выше работы относятся к случаю $d=2$. Первые результаты для $d=3$ появились в работе [31], где, следуя [18], доказаны теоремы существования решений начальной задачи для волн на водной поверхности в трех измерениях и, кроме того, приведены результаты о сходимости класса модельных уравнений длинных волн, которые связаны с приближением Буссинеска для случая $d=2$.

Проблема обоснования модуляционного предела тоже является важной задачей, где цель состоит в демонстрации того, что решения уравнений Эйлера, удовлетворяющие модуляционному анзацу, (i) существуют для достаточно длинного временного интервала и (ii) приближают решения нелинейного уравнения Шрёдингера. Предварительное исследование по этому вопросу при $d=2$ появилось в работе [32], а при $d=3$ в работе [33]. Однако в этих работах теорема существования для длинных интервалов времени не была доказана. Задача при $d=2$ была недавно решена Г. Шнайдером и Е. Вейном с частичным использованием преобразования нормальных форм для уравнений Эйлера (но не обязательно канонических преобразований). Задача строгого обоснования модуляционного приближения для размерности $d=3$ остается нерешенной.

ПроБЛЕмА 4. Обосновать различные модели волн для размерности $d=3$ как пределы уравнений поверхностных волн на воде. 
Другим значительным направлением исследований могло бы стать использование структуры уравнений волн на воде в виде системы Гамильтона для объяснения длинных волн и модуляционных масштабных режимов. Здесь могли бы быть задействованы преобразования нормальных форм и точный анализ, основанный на формальном подходе теории преобразования и разложения гамильтоновой системы по малому параметру, как это сделано в работах [34] и [35].

\section{5. Бегущие волны}

По данной теме обычными кандидатами для исследования являются два типа задач: или (i) задача с периодическими (по $x \in \mathbb{R}^{d-1}$ ) граничными условиями с некоторой периодической фундаментальной областью или (ii) задача об уединенных волнах. С использованием введенных ранее обозначений пространственно периодические решения удовлетворяют тождеству

$$
(\eta(x+\gamma, t), \xi(x+\gamma, t))=(\eta(x, t), \xi(x, t))
$$

при всех $\gamma \in \Gamma \subseteq \mathbb{R}^{d-1}$, где $\Gamma$ - периодическая решетка. Периодической фундаментальной областью служит тор $\mathbb{T}^{d-1}:=\mathbb{R}^{d-1} / \Gamma$. Решения типа бегущий волны удовлетворяют соотношению

$$
(\eta(x, t), \xi(x, t))=(\eta(x-t c), \xi(x-t c))
$$

для некоторого горизонтального вектора скорости $c \in \mathbb{R}^{d-1}$, и, следовательно, имеет место уравнение $\partial_{t}(\eta, \xi)=-c \cdot \partial_{x}(\eta, \xi)$. Понятие уединенной волны уже не столь определенно, и оно может означать разные объекты. Целям этого обзора лучше всего соответствуют решения уравнений типа бегущих волн, для которых $|\eta(x)| \rightarrow 0$ при $|x| \rightarrow+\infty$.

Исторический экскурс по этой тематике также состоит из нескольких сюжетов, представляющих независимые исследования в России и на Западе. А именно, первые результаты о существовании периодических бегущих волн на глубокой воде получены в работе А. И. Некрасова [20] в 1921 г., которая была совершенно неизвестна на Западе вплоть до 1967 г. Независимо Т. Леви-Чивита исследовал эту проблему в работе [19], опубликованной в 1925 г., где она была обобщена на случай постоянной фиксированной глубины водоема $b(x)=h$ в работе [21]. Силы поверхностного натяжения в расчет не принимались до работы [36] 1971 года.

Поскольку глубина водоема постоянна, задачу можно переформулировать в координатах, движущихся со скоростью решения, и в итоге получится эллиптическая задача с нелинейными граничными условиями на свободную поверхность. Чтобы справиться с границей, имеется много способов замены координат в этих уравнениях или с помощью конформных отображений (как у Леви-Чивита), или на основе лагранжевых координат, или с использованием более специфических методов. Литературные ссылки по этому вопросу очень разнообразны и всех их невозможно привести в одном коротком абзаце; мы ограничимся лишь следующими замечаниями.

Задача об уединенных волнах изучалась в [22] (1943 г.) и [23] (1954 г.). Глобальное рассмотрение этой задачи, которая по существу является нелинейной эллиптической задачей с бифуркацией, но со своими особенностями и сложностями, делалось в [37] и [38]. Последняя статья относится к так называемой "гипотезе Стокса" о наивысшей уединенной волне, или об уединенной волне 
“экстремальной формы”, а именно, о волне, которая имеет гребень с липшицевой особенностью с развернутым углом $2 \pi / 3$. В [39] и [40] были разработаны специальные методы для изучения уединенных волн с учетом и без учета поверхностного натяжения, и в них были получены содержательные ответы на вопросы об уединенных волнах с возможной асимптотической осцилляцией при $x \mapsto \pm \infty$.

В случае $d=3$ (или $d \geqslant 3$ ) поверхностное натяжение играет значительную роль. В [41] было показано, что в задаче с поверхностным натяжением существуют периодические волновые конфигурации типа "симметричный ромб". Совсем недавно в [42] была описана общая картина кратно-периодических решений. То, что при этом было обнаружено, связывает резонансное взаимодействие мод Фурье с кратностью решений нелинейной задачи. На самом деле, имеется тесная взаимосвязь между этой картиной и теоремой о резонансном центре Ляпунова в динамических системах, которая, в частности, применяется к гамильтоновым системам. В силу резонансного взаимодействия пятого порядка для конкретной фундаментальной области, существует два типа бегущих волн с разной геометрией - шестиугольные конфигурации и конфигурации серповидной формы. Форма шестиугольных структур зависит особым образом от глубины объема жидкости.

\section{ПроБЛЕма 5. Обгяснитъ природу серповидных волновых структур.}

Предыдущий абзац относится к конфигурациям бегущих волн с ненулевым поверхностным натяжением. Имеется серия замечательных результатов [43] и [44] о стоячих волнах для $d=2$, а недавно техника, разработанная в этих статьях, была использована применительно к задаче о трехмерных конфигурациях бегущих волн с поверхностным натяжением.

При $d=2$ (и без поверхностного натяжения) известно (см. [45]), что все уединенные волны имеют положительное возвышение, являются симметричными и монотонно убывают по обе стороны (единственного) гребня. В работе [38] о гипотезе Стокса доказано, что любая уединенная волна экстремальной формы имеет на гребне липшицеву особенность. Эта особенность также присутствует и в периодических конфигурациях бегущих волн, когда высота гребня достигает своего максимума, допустимого условием Бернулли. Уместно спросить об особенностях бегущих волн на свободной поверхности воды в случае $d=3$, когда они достигают максимально допустимой амплитуды.

Проблема 6. Какова природа особенностей на гребне экстремальных бегущих волн в трехмерном случае?

При $d=3$ (и без поверхностного натяжения) любая уединенная волна, которая убывает на бесконечности по пространственной переменной (напомним, что тогда $|\eta(x)| \rightarrow 0$ при $|x| \rightarrow+\infty)$, всегда является тривиальным решением $\eta=0$ (см. [46]). Таким образом, в трехмерном случае не существует неотрицательных уединенных волн.

ПроБлЕмА 7. Существуют ли нетривиальные трехмерные уединенные волны? Конечно, они должны быть знакопеременными.

Один вариант этой задачи связан с проблемой Ди Джоржи для нелинейного эллиптического уравнения с частными производными; а именно, требуется доказать, что трехмерное решение, которое стремится к нулю по одному направлению (т.е. имеется вектор $е$ такой, что для любого $x \quad|\eta(x+s e)| \rightarrow 0$ при $s \rightarrow \pm \infty)$, на самом деле является простым трехмерным продолжением двумерной уединенной волны (этот вариант задачи был предложен нашему вниманию 
Х. Брезисом). Если имеется поверхностное натяжение и число Бонда достаточно велико, то нетривиальные уединенные волны действительно существуют при $d=3$ (см. [47]); они являются уединенными волнами с вдавливанием.

$\mathrm{C}$ точки зрения практики большое значение имеет теория устойчивости разных конфигураций бегущих волн, и в математической литературе этому уделяется повышенное внимание. Работа [48] описывает взаимосвязь устойчивости уединенных волн при $d=2$ с вторичными бифуркациями главных ветвей решений.

Исключая уединенные волны, в океане почти невозможно встретить периодические волновые структуры, и кажется весьма вероятным, что большинство двоякопериодических решений (если не все) в трехмерном случае являются следствием линейной неустойчивости.

ПроБЛЕма 8. Построить теорию Блоха мод устойчивости и неустойчивости двоякопериодических конфигураций волн на воде.

Одно направление развития теории линейной устойчивости начинается с описания решений типа бегущей волны в следующих терминах. Для интегралов моментов $I_{j}(\eta, \xi)$ выполнены уравнения

$$
\partial_{x_{j}}\left(\begin{array}{l}
\eta \\
\xi
\end{array}\right)=J \operatorname{grad} I_{j} .
$$

Тогда в силу (5.2) решение системы (2.2) типа бегущей волны $\left(\left(\eta_{0}, \xi_{0}\right), c_{0}\right)$ будет удовлетворять равенству

$$
J(\operatorname{grad} H-c \cdot \operatorname{grad} I)=0 .
$$

Уравнение в вариациях для малого возмущения $v=(\delta \eta, \delta \xi)^{T}$, зависящего от времени, на волне $\left(\eta_{0}, \xi_{0}\right)$ имеет вид

$$
\partial_{t} v=J\left(\partial^{2} H\left(\eta_{0}, \xi_{0}\right)-c_{0} \cdot \partial^{2} I\left(\eta_{0}, \xi_{0}\right)\right) v .
$$

Линейная система (5.5) является автономной, поскольку она записана в координатах, движущихся с фазовой скоростью $c_{0}$, и поведение решений можно понять, изучая ассоциированную задачу на собственные значения

$$
\left(\partial^{2} H\left(\eta_{0}, \xi_{0}\right)-c_{0} \cdot \partial^{2} I\left(\eta_{0}, \xi_{0}\right)\right) V=-\lambda J V
$$

где следует обратить внимание на то, что правая часть этого уравнения содержит оператор $J$, дающий симплектическую форму. Блоховские граничные условия для $V=V(x, p)$ зависят от параметра $p \in \mathbb{R}^{d-1}$,

$$
V(x+\gamma, p)=e^{i p \cdot \gamma} V(x, p) \quad \forall \gamma \in \Gamma,
$$

что делает оператор в левой части уравнения (5.6) самосопряженным (для подходящей области определения). Пары собственная функция/собственный вектор $\left(V_{\ell}(x, p), \lambda_{\ell}(p)\right)$ из (5.6) зависят от $p$, а для их сдвигов $p \mapsto p+k$ выполнены равенства

$$
V_{\ell}(x+\gamma, p+k)=e^{i(p+k) \cdot \gamma} V_{\ell}(x, p+k)=e^{i p \cdot \gamma} V_{\ell}(x, p+k)
$$

при $k \in \Gamma^{\prime}$ из двойственной решетки (по определению двойственной решетки для любого $k \in \Gamma^{\prime}$ и для всех $\gamma \in \Gamma$ имеем $\left.\gamma \cdot k \in 2 \pi \mathbb{Z}\right)$. Иначе говоря, равенство $\left(V_{\ell}(x, p+k), \lambda_{\ell}(p+k)\right)=\left(V_{\ell^{\prime}}(x, p), \lambda_{\ell^{\prime}}(p)\right)$ выполнено для, возможно другого, 
индекса $\ell^{\prime}$, задаваемого в конечном счете оператором монодромии. По модулю этого свойства мы отождествляем параметры $k \in \Gamma^{\prime}$ и строим собственные функции и собственные векторы для двойственного тора $\left(\mathbb{T}^{d-1}\right)^{\prime}:=\mathbb{R}^{d-1} / \Gamma^{\prime}$ (зоны Бриллюена). По аналогией с теорией Блоха из квантовой механики, собственные значения $\lambda_{\ell}(p)$ принято называть функииями полосъ.

Если $\left(\eta_{0}, \xi_{0}\right)=0$, то функции $\lambda_{k}^{(0)}(p)=i\left(\left(c_{0} \cdot(k+p)\right) \pm \sqrt{g|k+p| \operatorname{th}(h|k+p|)}\right)$ естественно индексировать с помощью параметра $k \in \Gamma^{\prime}$. Их индекс Крейна ясен из выбора знака. Для фиксированного $p \in\left(\mathbb{T}^{d-1}\right)^{\prime}$ собственные величины могут оказаться кратными, когда $\lambda_{k}^{(0)}(p)=\lambda_{k^{\prime}}^{(0)}(p)$ для разных индексов $k, k^{\prime}$. При рассмотрении нетривиальных бегущих волн, скажем, из возмущенного семейства для нулевого решения, собственные величины $\lambda_{k}(p)$ будут меняться, и точки $p$, для которых собственные значения $\lambda_{k}^{(0)}(p)$ были кратными и имели противоположные сигнатуры Крейна, могут бифурцировать, приобретая положительную вещественную часть; при этом образуется неустойчивая область. Это вполне соответствует картине из теории Блоха для оператора Шрёдингера, за тем исключением, что оператор $J$ в (5.6) отличается от тождественного оператора $I$ и задача становится инфинитезимально симплектической, а не самосопряженной. Отказываясь от свойства вещественности собственных значений, получаем следующее правило: если $\lambda_{k}(p)$ лежит на спектре задачи (5.6), то точки $-\lambda_{k}(p)$ и $\pm \overline{\lambda_{k}(p)}$ также принадлежат спектру и, следовательно, неустойчивые собственные значения появляются или парами, или целыми четверками. Полная теория Блоха описала бы области нестабильности и дала бы согласованную индексацию для пар собственная функция/собственное значение $\left(V_{\ell}(x, p), \lambda_{\ell}(p)\right)$, которые очевидно определены на ветвях, покрывающих тор, как и правила перехода для монодромии отображений $\ell \mapsto \ell^{\prime}$ при $p \mapsto p+k$ для $k \in \Gamma^{\prime}$.

Возвращаясь к теме уединенных волн, было бы полезно иметь строгую теорию устойчивости уединенных волн, а при $d=2$ стоит также задаться вопросом об их устойчивости под действием трехмерных возмущений.

\section{6. Инвариантные структуры в фазовом пространстве}

Линеаризация системы уравнений (2.2) делается весьма элегантным способом с помощью разложения Тейлора гамильтониана (2.4) с сохранением только квадратичных по $(\eta, \xi)$ членов. Ограничимся рассмотрением задачи с периодическими граничными условиями: $x \in \mathbb{R}^{d-1} / \Gamma:=\mathbb{T}^{d-1}$, где $\Gamma \subseteq \mathbb{R}^{d-1}$ - заданная решетка трансляций. Квадратичная часть рассматриваемого гамильтониана равна

$$
H^{(2)}(\eta, \xi)=\int_{\mathbb{T}^{d-1}}\left(\frac{1}{2} \xi(x) G(0) \xi(x)+\frac{g}{2} \eta^{2}(x)\right) d x,
$$

что с помощью тождества Планшереля можно переписать в терминах преобразования Фурье (канонического преобразования) и коэффициентов Фурье оператора Дирихле-Неймана в виде

$$
H^{(2)}(\eta, \xi)=\sum_{k \in \Gamma^{\prime}}\left(\frac{1}{2}|k| \operatorname{th}(h|k|)|\widehat{\xi}(k)|^{2}+\frac{g}{2}|\widehat{\eta}(k)|^{2}\right) .
$$

Уравнения Гамильтона кратко запишутся в виде

$$
\partial_{t}\left(\begin{array}{l}
\eta \\
\xi
\end{array}\right)=\left(\begin{array}{cc}
0 & I \\
-I & 0
\end{array}\right)\left(\begin{array}{l}
\partial_{\eta} H^{(2)} \\
\partial_{\xi} H^{(2)}
\end{array}\right)
$$


и, в частности,

$$
\partial_{t} \widehat{\eta}(k, t)=|k| \operatorname{th}(h|k|) \widehat{\xi}(k, t), \quad \partial_{t} \widehat{\xi}(k, t)=-g \widehat{\eta}(k, t),
$$

а решения этих уравнений образуют линейный поток

$$
\Phi_{t}^{0}(\eta, \xi)=\sum_{k \in \Gamma^{\prime}}\left(\begin{array}{cc}
\cos (\omega(k) t) & \sin (\omega(k) t) / \omega(k) \\
-\omega(k) \sin (\omega(k) t) & \cos (\omega(k) t)
\end{array}\right)\left(\begin{array}{l}
\widehat{\eta}(k) \\
\widehat{\xi}(k)
\end{array}\right),
$$

где $\omega(k)=\sqrt{g|k| \operatorname{th}(h|k|)}$. Ясно, что все решения (возможно, за исключением $\widehat{\xi}(0, t))$ этих линейных уравнений эволюционируют на инвариантных торах в фазовом пространстве, которые получаются замыканием их орбит: $\mathbb{T}^{m}=$ $\overline{\left\{\Phi_{t}^{0}(\eta(x), \xi(x)): t \in \mathbb{R}\right\}}$. Тогда можно сказать, что все решения линеаризованных уравнений являются периодическими (при $m=1$ получаются инвариантные окружности в фазовом пространстве), квазипериодическими (для случая $m<+\infty)$ или почти периодическими $(m=\infty)$ функциями времени. Резонансные торы порождают параметрические семейства таких решений, а сами торы параметризуются с помощью переменных $I(k)=(\omega(k) / 2 g)|\widehat{\eta}(k)|^{2}+$ $(g / 2 \omega(k))|\widehat{\xi}(k)|^{2}$. Возникает естественный вопрос, будет ли любое решение нелинейных уравнений удовлетворять этим сильным свойствам рекуррентности. По аналогии с гамильтоновыми системами следует ожидать, что теория возмущений квазипериодических решений приведет к задаче с малыми знаменателями. Это тем более верно для почти периодических решений. Однако, поскольку мы изучаем уравнения с частными производными, задача с малыми знаменателями возникает также и для периодических решений.

В двумерном случае $(d=2)$ недавно наблюдался прогресс в задаче с периодическими по времени решениями в форме стоячих волн - см. [43] для случая водоема конечной глубины и [44] для случая $h=+\infty$. Обе эти статьи используют некоторый вариант схемы Нэша-Мозера, чтобы справиться с проблемой малых знаменателей.

ПроБлемА 9. Доказать существование параметрических семейств квазипериодических и/ или почти периодических решений задачи о волнах на поверхности води.

Эта задача включает случай периодических по времени решений в пространстве более высокой размерности. Простой вариант периодического решения представляет собой пространственно периодическую бегущую волну; чтобы избавиться от этого простого решения, можно поставить граничные условия Неймана по $x \in \mathbb{R}$ (стоячие волны), что было сделано в работах [43] и [44]. Можно также искать условия, гарантирующие, что найденные решения устроены более сложно, чем те, которые являются инвариантными в движущейся системе координат. Можно надеяться обнаружить семейства решений с канторовым параметром, которые являются гладкими по Уитни, но не гладкими в классическом смысле. Такие решения существуют в случае конечномерных гамильтоновых систем в присутствии малых знаменателей.

Рассмотрим теперь задачу для $d=2$ в некомпактной постановке при $x \in \mathbb{R}^{1}$. Имеется интересный вопрос о взаимодействии уединенных волн. Для особых уравнений типа $\mathrm{KdV}$ в дополнение к точным солитонам имеется специальный класс кратно-солитонных решений, которые являются асимптотиками конечного числа уединенных волн при $t \mapsto \pm \infty$ и которые взаимодействуют друг с другом при встрече простым сложением. Однако они являются решениями модельных уравнений. Ожидается, что решения типа уединенной волны для 


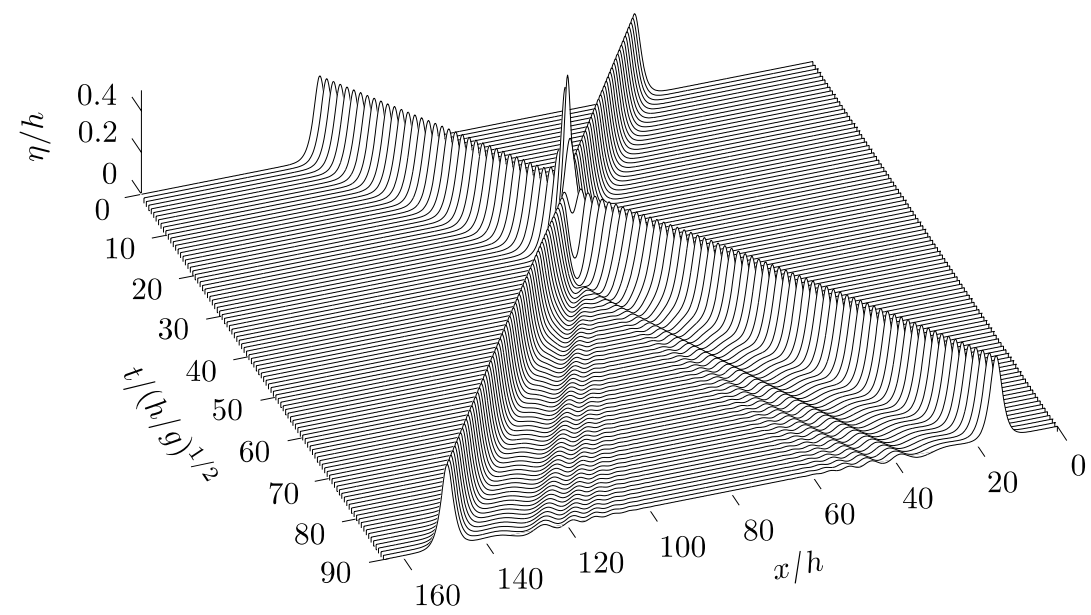

Рис. 1. Встречное столкновение двух уединенных волн одинаковой высоты $S / h=0.4$, демонстрирующее разбегание волн, их задержку по фазе и рассеивающийся хвост, порожденные при взаимодействии. Амплитуда уединенных волн после столкновения немного меньше, чем до столкновения: в момент времени $t / \sqrt{h / g}=90$ ее величина равна $S^{+} / h=0.3976$. Кроме того, результирующие уединенные волны немного отстают от своих линейных траекторий, прошедших без столкновения. Это отставание по фазе равно $\left(a_{j}-a_{j}^{+}\right) / h=0.3257$, где $a_{j}$ (соответственно $\left.a_{j}^{+}\right)$- отсечки по оси $t$ асимптотически линейных траекторий до (соответственно после) столкновения.

задачи волн на воде не будут взаимодействовать так просто, однако важно знать, насколько мал будет корректирующий остаток после столкновения волн в зависимости от их амплитуды. Численные и экспериментальные результаты из работы [49] говорят, что уединенные волны на воде, по всей видимости, являются устойчивыми при умеренных амплитудных режимах, и наблюдается следующий результат столкновения двух уединенных волн: сами исходные решения превращаются в две слегка трансформированные уединенные волны плюс малый импульсный остаток, после чего все три компоненты разделяются, и дальнейшая их эволюция происходит независимо друг от друга, см. результат численного моделирования на рис. 1 . В общем случае можно предполагать, что любые локализованные начальные данные задачи о свободных волнах на воде распадаются через некоторое (достаточно долгое) время на две последовательности уединенных волн (бегущих влево и вправо), которые двигаются впереди рассеивающейся остаточной компоненты.

ПроБЛЕма 10. Доказать, что имеет место нарисованная выше картина рассеивания. Иначе говоря, общие начальные данные, имеющие определенную локализацию, распадаются на две серии уединенных волн плюс остаток, причем этот процесс описывается оператором рассеяния, связанным с линейной задачей.

Все это указывает на то, что при больших временах решения определяются (i) конечным числом параметров (амплитудами и фазами асимптотических 
уединенных волн) и (ii) линейным оператором рассеяния для остатка. В настоящий момент даже не известно, существует ли решение задачи поверхностных волн в форме суперпозиции из $N$ уединенных волн, имеющих разную скорость на временном интервале $t \in[0,+\infty)$ и асимптотически разделяющихся при $t \rightarrow+\infty$ (эту задачу сформулировали в 1979 г. Ю. Мозер и Р. Сакс).

В том случае, когда нарисованная выше картина рассеивания не выполняется, основные законы сохранения для задачи о волнах на воде дают базовые соотношения (см. [49]) между входящими и выходящими уединенными волнами вместе с массой, моментом и энергией остатка. Точные решения типа уединенной волны возникают в однопараметрическом семействе $\left(\eta_{s}(x), \xi_{s}(x), c_{s}\right)$, которое параметризуется своей амплитудой $s:=\left\|\eta_{s}\right\|_{L^{\infty}}$ (следуя Стоксу). На большей части параметрического диапазона имеется единственное решение для каждого значения параметра $s$ и для каждого направления распространения (однако вопрос единственности не полностью решен для экстремальных точек параметрического диапазона). Это семейство решений имеет четко заданные значения для трех постоянных величин, а именно для массы $m(s)=M\left(\eta_{s}\right)$, момента $i(s)=I\left(\eta_{s}, \xi_{s}\right)$ и энергии $e(s)=H\left(\eta_{s}, \xi_{s}\right)$. Уединенная волна, перемещающаяся слева направо, имеет положительные массу, момент и энергию, а идентичная ей волна, двигающаяся справа налево, имеет постоянные массу, момент и энергию с теми же модулями, но со знаком минус. Рассматриваемая ситуация состоит в том, что решение стремится к двум асимптотически разделяющимся уединенным волнам $\eta_{s_{1}}, \eta_{s_{2}}$ при $t \rightarrow-\infty$, а именно,

$$
\lim _{t \rightarrow-\infty}\left\|(\eta(x, t), \xi(x, t))-\left(\eta_{s_{1}}, \xi_{s_{1}}\right)\left(x-t c_{s_{1}}\right)-\left(\eta_{s_{2}}, \xi_{s_{2}}\right)\left(x-t c_{s_{2}}\right)\right\|_{L^{\infty}}=0 .
$$

Может случиться, что скорости $c_{s_{1}}$ и $c_{s_{2}}$ имеют противоположные знаки, и в этом случае изучается встречное столкновение уединенных волн, а если они имеют одинаковый знак, то исследуется случай попутного столкновения. Из соотношения (6.6) можно найти общую массу, момент и энергию этого решения при $t \rightarrow-\infty$, которые равны соответственно

$$
M_{T}=m\left(s_{1}\right)+m\left(s_{2}\right), \quad I_{T}=i\left(s_{1}\right)+i\left(s_{2}\right), \quad E_{T}=e\left(s_{1}\right)+e\left(s_{2}\right) .
$$

Теперь, взяв большое положительное время, исследуем решение после взаимодействия. В силу картины рассеивания решение $(\eta(x, t), \xi(x, t))$ раскладывается на три компоненты: две уединенные волны $\left(\eta_{s_{1}^{+}}, \xi_{s_{1}^{+}}\right)\left(x-t c_{s_{1}^{+}}-a_{1}\right)$, $\left(\eta_{s_{2}^{+}}, \xi_{s_{2}^{+}}\right)\left(x-t c_{s_{2}^{+}}-a_{2}\right)$ и корректирующий остаток $\left(\eta_{R}(x, t), \xi_{R}(x, t)\right)$. Параметры $a_{1}, a_{2}$ представляют сдвиг по фазе решений в результате взаимодействия. Три постоянные величины можно вычислить также после взаимодействия. Сделаем это через достаточно большой промежуток времени, когда две уединенные волны разойдутся на большое расстояние друг от друга и от остатка $\left(\eta_{R}, \xi_{R}\right)$, так что

$$
M_{T}=m\left(s_{1}^{+}\right)+m\left(s_{2}^{+}\right)+m_{R}, \quad I_{T}=i\left(s_{1}^{+}\right)+i\left(s_{2}^{+}\right)+i_{R}, \quad E_{T}=e\left(s_{1}^{+}\right)+e\left(s_{2}^{+}\right)+e_{R},
$$

где $m_{R}, i_{R}$ и $e_{R}$ - масса, момент и энергия остатка. Взяв соответствующие разности между (6.7) и (6.8), получаем

$$
\begin{aligned}
\left(m\left(s_{1}\right)-m\left(s_{1}^{+}\right)\right)+\left(m\left(s_{2}\right)-m\left(s_{2}^{+}\right)\right) & =m_{R}, \\
\left(i\left(s_{1}\right)-i\left(s_{1}^{+}\right)\right)+\left(i\left(s_{2}\right)-i\left(s_{2}^{+}\right)\right) & =i_{R}, \\
\left(e\left(s_{1}\right)-e\left(s_{1}^{+}\right)\right)+\left(e\left(s_{2}\right)-e\left(s_{2}^{+}\right)\right) & =e_{R} .
\end{aligned}
$$


Обозначим $\delta s_{1}=s_{1}-s_{1}^{+}$и $\delta s_{2}=s_{2}-s_{2}^{+}$. В силу теоремы о промежуточном значении, имеются промежуточные точки $s_{j}(*)$, где $j=1,2$ и $*=m, i$ и $e$, такие, что

$$
\begin{aligned}
m\left(s_{j}\right)-m\left(s_{j}^{+}\right) & =m^{\prime}\left(s_{j}(m)\right) \delta s_{j}, \\
i\left(s_{j}\right)-i\left(s_{j}^{+}\right) & =i^{\prime}\left(s_{j}(i)\right) \delta s_{j}, \\
e\left(s_{j}\right)-e\left(s_{j}^{+}\right) & =e^{\prime}\left(s_{j}(e)\right) \delta s_{j} .
\end{aligned}
$$

В общем случае изменения амплитуды в результате одного из этих взаимодействий уединенных волн являются удивительно малыми даже для достаточно больших значений амплитуды. Поэтому $s_{j}(*)$ будет хорошо аппроксимироваться величинами $s_{j}, j=1,2$, которые определяют начальные данные. Используя (6.10) в уравнении (6.9), получаем три уравнения для двух неизвестных $\delta s_{1}$ и $\delta s_{2}$, что влечет определенные соотношения между коэффициентами (6.9). Эти соотношения представляют собой ограничения на массу, момент и энергию, которые могут перейти к остатку при столкновении. В частности, в случае симметричного встречного столкновения $I_{T}=0$ и $\delta s_{1}=\delta s_{2}$, а из уравнений $(6.9)$ и (6.10) вытекает, что $i_{R}=0$, т.е.

$$
e_{R}=\frac{e^{\prime}(s)}{m^{\prime}(s)} m_{R}
$$

Поскольку $e_{R} \geqslant 0$, из уравнений (6.9) и (6.10) следует, что $\delta s \geqslant 0$, а также что $m_{R} \geqslant 0$. Предположим, что остаток лежит в промежутке между двумя уединенными волнами, появившимися после столкновения (это является частью картины рассеивания), и его длина равна $2 L$ через достаточно большое время $t$ после взаимодействия. Поскольку $m_{R}=\int \eta_{R} d x \leqslant \sqrt{2 L}\left\|\eta_{R}\right\|_{L^{2}}$ и $\left\|\eta_{R}\right\|_{L^{2}}^{2} \leqslant(2 / g) e_{R}$, получаем следующую оценку для энергии остатка:

$$
e_{R} \leqslant \frac{e^{\prime}(s)}{m^{\prime}(s)} m_{R} \leqslant \frac{e^{\prime}(s)}{m^{\prime}(s)} \sqrt{\frac{4 L}{g} e_{R}} .
$$

Из этого неравенства, в частности, следует оценка сверху для энергии, которая может перейти остатку после столкновения:

$$
e_{R} \leqslant \frac{4 L}{g}\left(\frac{e^{\prime}}{m^{\prime}}\right)^{2} .
$$

Аналогичное неравенство имеет место для случая, когда амплитуды входящих уединенных волн различны. Если встречное столкновение не является симметричным, то из приведенных выше рассуждений нельзя извлечь объяснение для возможного изменения знака разности амплитуд $\delta s_{j}$ (хотя, конечно, обе они не могут быть одновременно отрицательными, поскольку $\left.e_{R} \geqslant 0\right)$. Тем не менее численные и экспериментальные данные указывают на то, что (1) при встречных столкновениях обе разности $\delta s_{j}>0$, и (2) при попутных столкновениях, когда волна с большей амплитудой $\eta_{s_{1}}$ догоняет волну с меньшей амплитудой $\eta_{s_{2}}$, всегда $\delta s_{1}<0$ и $\delta s_{2}>0$. Таким образом, похоже, что встречное столкновение приводит к некоторому уменьшению амплитуды сталкивающихся волн, а попутное столкновение приводит к усилению большей волны и к ослаблению меньшей. Изменения амплитуд малы даже для больших величин амплитуд сталкивающихся уединенных волн. Кроме того, при попутном 
столкновении амплитуды меняются очень мало, и, возможно, величины их изменения на порядок меньше, чем при встречном столкновении волн с такими же амплитудами.

При сделанных предположениях из уравнений (6.9), (6.10) можно вывести несколько дальнейших следствий. Первое наблюдение состоит в том, что

$$
0 \leqslant e_{R}=e^{\prime}\left(s_{1}\right) \delta s_{1}+e^{\prime}\left(s_{2}\right) \delta s_{2},
$$

где взято $s_{1} \geqslant s_{2}$ и предполагается $\delta s_{1}=s_{1}-s_{1}^{+} \leqslant 0$, откуда следует, что $\delta s_{2}=$ $s_{2}-s_{2}^{+} \geqslant 0$. Однако известно, что энергия $e(s)$ уединенной волны является возрастающей выпуклой функцией аргумента $s$, и поэтому из (6.14) находим, что

$$
\frac{s_{1}^{+}-s_{1}}{s_{2}-s_{2}^{+}} \leqslant \frac{e^{\prime}\left(s_{2}\right)}{e^{\prime}\left(s_{1}\right)} \leqslant 1 .
$$

В частности, $s_{1}^{+}-s_{1} \leqslant s_{2}-s_{2}^{+}$. Это означает, что более высокая уединенная волна не может получить прирост амплитуды больше потери амплитуды низкой волны. Второе заключение относится к массе $m_{R}$ остатка (и оно, на самом деле, вытекает из нашего первого наблюдения). Интеграл массы не имеет априорного знака, но

$$
m_{R}=m^{\prime}\left(s_{1}\right) \delta s_{1}+m^{\prime}\left(s_{2}\right) \delta s_{2},
$$

где $-\delta s_{2} \leqslant \delta s_{1} \leqslant 0 \leqslant \delta s_{2}$. Поскольку известно, что $m(s)$ является возрастающей выпуклой функцией переменной $s$ почти на всем диапазоне амплитуд $s$, где существует уединенная волна, получаем, что $m^{\prime}\left(s_{1}\right) \leqslant m^{\prime}\left(s_{2}\right)$, откуда следует неравенство $\left|m^{\prime}\left(s_{1}\right) \delta s_{1}\right| \leqslant m^{\prime}\left(s_{2}\right) \delta s_{2}$ и, значит,

$$
m_{R} \leqslant 0 \text {. }
$$

На самом деле, в этом поведении наблюдается тенденция уединенных волн на поверхности воды к весьма слабому затуханию при попутных столкновениях.

\section{7. Волновая турбулентность}

Детальное исследование динамики индивидуальных волн на поверхности воды является весьма полезной задачей, однако изучение тонкой структуры волновых полей в масштабах океана является практически неосуществимым как с численной, так и теоретической точки зрения. Тем не менее, предсказание этих явлений природы остается чрезвычайно важной задачей прогнозирования состояния океана, которое весьма актуально в мореплавании. Подход к количественному анализу поведения ряда макроскопических величин, связанный с эволюцией волн в океане, приводит к процессам усреднения, которые описываются теоретико-подобными эволюционными уравнениями для этих макроскопических величин. Такие приближенные процессы заслуживают рассмотрения на строгом математическом уровне. В этом плане имеется два теоретических построения в работах [50] и [51], [52]; первое является в некотором смысле специализированнъм, а второе основано на физических рассмотрениях и на гамильтоновом формализме при написании уравнений движения. Для представления нашей собственной точки зрения запишем канонические переменные задачи волн на воде в комплексных симплектических координатах в виде

$$
a(x):=\sqrt{\frac{g}{2 \omega(D)}} \eta(x)+i \sqrt{\frac{\omega(D)}{2 g}} \xi(x),
$$


где $D=-i \partial_{x}$. При этом преобразовании квадратичный гамильтониан (полученный при линеаризации уравнений движения) равен

$$
H^{(2)}=\int \bar{a}(x)(\omega(D) a(x)) d x,
$$

что соответствует (6.4). Следуя по пути работы [51] и работая на формальном математическом уровне, делаем преобразования нормальных форм гамильтониана (2.4) до некоторого порядка (скажем, $N=4$ ). Обозначим новые переменные через $b=\tau_{N}(a)$ и рассмотрим их преобразование Вигнера

$$
W_{\varepsilon}[b](x, k, t):=\frac{1}{\varepsilon^{d-1}} \int_{\mathbb{R}_{y}^{d-1}} e^{i k \cdot y} \bar{b}(x-y / \varepsilon) b(x+y / \varepsilon) d y .
$$

С точностью до более высоких степеней $\varepsilon$, преобразование Вигнера $W=W_{\varepsilon}[b]$ будет удовлетворять уравнению, похожему на уравнение Больцмана ([53] и рассмотрения К. Бардоса, В. Крейга и В. Панферова)

$$
\partial_{t} W+\partial_{k} \omega(k) \cdot \partial_{x} W=S_{n l},
$$

где нелинейный член взаимодействия $S_{n l}$ выводится из квадратичных членов и членов более высокого порядка гамильтониана $H$ задачи волн на воде в разложении его нормальной формы. Их самих надо выразить в терминах преобразования Вигнера, что является одной из причин для использования нормальной формы. В этом месте часто берется среднее от $W$ по статистическому ансамблю волновых полей, возможно, при некоторых предположениях на замыкание случайных фаз. Все эти манипуляции являются достаточно формальными.

ПроблемА 11. Обосновать этот подход в свете кинетической теории распространения и взаимодействия волновых полей. Дать возможно более строгий математический вывод для уравнения (7.3).

Математический анализ преобразования нормальных форм, который неявно присутствует в приведенной выше программе исследований, сам по себе является хорошей задачей.

Подобно тому как стационарные распределения в операторе столкновения (распределение Максвелла) играют особую роль для уравнения Больцмана, стационарные распределения для $S_{n l}$ должны играть важную роль в теории решений уравнения (7.3). В самом деле, их следовало бы параметризовать с помощью основных макроскопических переменных задачи, чья динамика будет определяться некоторыми макроскопическими уравнениями движения. В случае однородных по $x \in \mathbb{R}^{d-1}$ волновых полей решения для $S_{n l}=0$ в форме степенного закона были найдены В.Е. Захаровым и его соавторами, поэтому будет правильным называть эти решения спектром Колмогорова-Захарова. Экспериментальное подтверждение для спектрального поведения усредненных волновых полей было впервые получено на озере Онтарио (см. [54]). По аналогии с распределениями Максвелла в теории уравнения Больцмана параметрические семейства такого спектра приводят к определению макроскопических переменных, которые меняются в зависимости от переменных $(x, t)$ физического пространства-времени при решении уже неоднородной задачи. Преобразование Вигнера $W(x, k, t)$ по существу является микролокализацией волнового поля, заданного с помощью $b(x, t)$. Однако распределения по степенному закону могут быть не единственными однородными волновыми полями, которые служат решениями для $S_{n l}=0$, и, возможно, имеются другие параметрические семейства решений для разных нелинейных режимов. Всевозможные 
параметрические семейства таких решений могут породить разные множества макроскопических переменных для разных режимов. Это приводит нас к формулировке последней проблемы.

ПроБЛЕмА 12. Доказать, что существуют спектры, удовлетворяющие степенному закону Колмогорова-Захарова, и они управляют асимптотиками волновых полей для больших времен, по крайней мере в некоторых конкретных ситуациях. Найти полное множество решений для $S_{n l}=0$, а также их соответствующие переменные, и вывести подходящие макроскопические уравнения, которые описывают их эволющию.

\section{Список литературы}

[1] V.E. Zakharov, "Stability of periodic waves of finite amplitude on the surface of a deep fluid", J. Appl. Mech. Tech. Phys., 9:2 (1968), 190-194.

[2] W. Craig, C. Sulem, "Numerical simulation of gravity waves", J. Comput. Phys., 108:1 (1993), 73-83.

[3] J. C. Luke, "A variational principle for a fluid with a free surface", J. Fluid Mech., 27 (1967), 395-397.

[4] L. V. Ovsiannikov, "Non local Cauchy problems in fluid dynamics", Actes du congrès international des mathématiciens, vol. 3 (Nice, 1970), Gauthier-Villars, Paris, 1971, 137-142.

[5] В. И. Налимов, “Задача Коши-Пуассона”, Динамика сплошной средь, 18 (1974), 104-210.

[6] T. Kano, T. Nishida, "Sur les ondes de surface de l'eau avec une justification mathématique des équations des ondes en eau peu profonde", J. Math. Kyoto Univ., 19:2 (1979), 335-370.

[7] M. Shinbrot, "The initial value problem for surface waves under gravity. I. The simplest case", Indiana Univ. Math. J., 25:3 (1976), 281-300.

[8] J. Reeder, M. Shinbrot, "The initial value problem for surface waves under gravity. II. The simplest 3-dimensional case", Indiana Univ. Math. J., 25:11 (1976), 1049-1071.

[9] J. Reeder, M. Shinbrot, "The initial value problem for surface waves under gravity. III. Uniformly analytic initial domains", J. Math. Anal. Appl., 67:2 (1979), 340-391.

[10] C. Sulem, P.-L. Sulem, C. Bardos, U. Frisch, "Finite time analyticity for the two- and three-dimensional Kelvin-Helmholtz instability", Comm. Math. Phys., 80:4 (1981), 485-516.

[11] H. Yosihara, "Gravity waves on the free surface of an incompressible perfect fluid of finite depth", Publ. Res. Inst. Math. Sci., 18:1 (1982), 49-96.

[12] H. Yosihara, "Capillary-gravity waves for an incompressible ideal fluid", J. Math. Kyoto Univ., 23:4 (1983), 649-694.

[13] W. Craig, "An existence theory for water waves and the Boussinesq and Korteweg-de Vries scaling limits", Comm. Partial Differential Equations, 10:8 (1985), 787-1003.

[14] M. Shinbrot, C. E. Wayne, "The long-wave limit for the water wave problem. I. The case of zero surface tension", Comm. Pure Appl. Math., 53:12 (2000), 1475-1535.

[15] M. Shinbrot, C. E. Wayne, "The rigorous approximation of long-wavelength capillarygravity waves", Arch. Ration. Mech. Anal., 162:3 (2002), 247-285.

[16] S. Wu, "Well-posedness in Sobolev spaces of the full water wave problem in 2-D", Invent. Math., 130:1 (1997), 39-72.

[17] S. Wu, "Well-posedness in Sobolev spaces of the full water wave problem in 3-D", J. Amer. Math. Soc., 12:2 (1999), 445-495. 
[18] D. Lannes, "Well-posedness of the water-waves equations", J. Amer. Math. Soc., 18:3 (2005), 605-654.

[19] T. Levi-Civita, "Détermination rigoureuse des ondes permanentes d'ampleur finie", Math. Ann., 93:1 (1925), 264-314.

[20] A.I. Nekrasov, The exact theory of steady state waves on the surface of a heavy liquid, MRC Technical Report № 813, Univ. of Wisconsin, Madison, 1967.

[21] D. Struik, "Détermination rigoureuse des ondes irrotationnelles périodiques dans un canal à profondeur finie", Math. Ann., 95:1 (1926), 595-634.

[22] М. А. Лаврентьев, "К теории длинных волн", Докл. АН СССР, 41:7 (1943), 289291; англ. пер.: M. A. Lavrentieff, "A contribution to the theory of long waves", $C$. R. (Doklady) Acad. Sci. URSS (N. S.), 41 (1943), 275-277.

[23] K. O. Friedrichs, D. H. Hyers, "The existence of solitary waves", Comm. Pure Appl. Math., 7:3 (1954), 517-550.

[24] D. Christodoulou, H. Lindblad, "On the motion of the free surface of a liquid", Comm. Pure Appl. Math., 53:12 (2000), 1536-1602.

[25] H. Lindblad, "Well-posedness for the motion of an incompressible liquid with free surface boundary", Ann. of Math. (2), 162:1 (2005), 109-194.

[26] D. Ebin, "The equations of motion of a perfect fluid with free boundary are not well posed", Comm. Partial Differential Equations, 12:10 (1987), 1175-1201.

[27] T. B. Benjamin, P. J. Olver, "Hamiltonian structure, symmetries and conservation laws for water waves", J. Fluid Mech., 125 (1982), 137-185.

[28] T. Kano, T. Nishida, "Water waves and Friedrichs expansion", Recent topics in nonlinear PDE (Hiroshima, 1983), North-Holland Math. Stud., 98, North-Holland, Amsterdam, 1984, 39-57.

[29] T. Kano, T. Nishida, "A mathematical justification for Korteweg-de Vries equation and Boussinesq equation of water surface waves", Osaka J. Math., 23:2 (1986), 389-413.

[30] J.D. Wright, "Corrections to the KdV approximation for water waves", SIAM J. Math. Anal., 37:4 (2005), 1161-1206.

[31] J. Bona, Th. Colin, D. Lannes, "Long wave approximations for water waves", Arch. Ration. Mech. Anal., 178:3 (2005), 373-410.

[32] W. Craig, C. Sulem, P.-L. Sulem, "Nonlinear modulation of gravity waves: a rigorous approach", Nonlinearity, 5:2 (1992), 497-522.

[33] W. Craig, U. Schanz, C. Sulem, "The modulational regime of three-dimensional water waves and the Davey-Stewartson system", Ann. Inst. H. Poincaré Anal. Non Linéaire, 14:5 (1997), 615-667.

[34] W. Craig, M. Groves, "Hamiltonian long-wave approximations to the water-wave problem", Wave Motion, 19:4 (1994), 367-389.

[35] W. Craig, P. Guyenne, D. Nicholls, C. Sulem, "Hamiltonian long-wave expansions for water waves over a rough bottom", Proc. R. Soc. Lond. Ser. A. Math. Phys. Eng. Sci., 461:2055 (2005), 839-873.

[36] E. Zeidler, "Existenzbeweis für cnoidal waves unter Berücksichtigung der Oberflächenspannung", Arch. Rational Mech. Anal., 41:2 (1971), 81-107.

[37] C. J. Amick, J. F. Toland, "On solitary water-waves of finite amplitude", Arch. Ration. Mech. Anal., 76:1 (1981), 9-95.

[38] C. J. Amick, L.E. Fraenkel, J.F. Toland, "On the Stokes conjecture for the wave of extreme form", Acta Math., 148:1 (1982), 193-214.

[39] K. Kirchgässner, "Nonlinear wave motion and homoclinic bifurcation", Theoretical and applied mechanics (Lyngby, 1984), North-Holland, Amsterdam, 1985, 219-231.

[40] A. Mielke, Hamiltonian and Lagrangian flows on center manifolds. With applications to elliptic variational problems, Lecture Notes in Math., 1489, Springer-Verlag, Berlin, 1991. 
[41] J. Reeder, M. Shinbrot, "Three-dimensional, nonlinear wave interaction in water of constant depth", Nonlinear Anal., 5:3 (1981), 303-323.

[42] W. Craig, D. Nicholls, "Travelling two and three dimensional capillary gravity water waves", SIAM J. Math. Anal., 32:2 (2000), 323-359.

[43] P. I. Plotnikov, J. F. Toland, "Nash-Moser theory for standing water waves", Arch. Ration. Mech. Anal., 159:1 (2001), 1-83.

[44] G. Iooss, P. I. Plotnikov, J. F. Toland, "Standing waves on an infinitely deep perfect fluid under gravity", Arch. Ration. Mech. Anal., 177:3 (2005), 367-478.

[45] W. Craig, P. Sternberg, "Symmetry of solitary waves", Comm. Partial Differential Equations, 13:5 (1988), 603-633.

[46] W. Craig, "Non-existence of solitary water waves in three dimensions", R. Soc. Lond. Philos. Trans. Ser. A. Math. Phys. Eng. Sci., 360:1799 (2002), 2127-2135.

[47] M. Groves, S.-M. Sun, "Fully localised solitary-wave solutions of the three-dimensional gravity-capillary water-wave problem", Arch. Ration. Mech. Anal (to appear).

[48] П.И. Плотников, "Неединственность решений задачи об уединенных волнах и бифуркации критических точек гладких функционалов", Изв. РАН. Сер. матем., 55:2 (1991), 339-366; англ. пер.: P. I. Plotnikov, "Nonuniqueness of solutions of a problem on solitary waves, and bifurcations of critical points of smooth functionals", Math. USSR-Izv., 38:2 (1992), 333-357.

[49] W. Craig, P. Guyenne, J. Hammack, D. Henderson, C. Sulem, "Solitary water wave interactons", Phys. Fluids, 18:5 (2006), 057106.

[50] K. Hasselmann, "On the non-linear energy transfer in a gravity-wave spectrum. I. General theory", J. Fluid Mech., 12 (1962), 481-500.

[51] В.Е. Захаров, В.С. Львов, "Статистическое описание нелинейных волновых полей”, Изв. вузов. Сер. радиофиз., 18:10 (1975), 1470-1487; англ. пер.: V.E. Zakharov, V.S. L'vov, "Statistical description of nonlinear wave fields", Radiophys. and Quantum Electronics, 18:10 (1975), 1084-1097.

[52] V.E. Zakharov, V.S. L'vov, G. Falkovich, Kolmogorov spectra of turbulence. I. Wave turbulence, Springer-Verlag, Berlin-New York, 1992.

[53] P. Gérard, P. Markowich, N. Mauser, F. Poupaud, "Homogenization limits and Wigner transforms", Comm. Pure Appl. Math., 50:4 (1997), 323-379.

[54] M. A. Donelan, J. Hamilton, W.H. Hui, "Directional spectra of wind-generated waves", R. Soc. Lond. Philos. Trans. Ser. A. Math. Phys. Eng. Sci., 315:1534 (1985), $509-562$.

\section{В. Крейг (W. Craig)}

McMaster University, Hamilton, Canada

E-mail: craig@math.mcmaster.ca

\section{К. Е. Вейн (С. Е. Wayne)}

Department of Mathematics

Boston University

Boston, USA

E-mail: cew@math.bu.edu 